\title{
Thermally induced magnetic relaxation in building blocks of artificial kagome spin ice
}

\author{
Alan Farhan, ${ }^{1,2}$ Armin Kleibert,,${ }^{3, *}$ Peter M. Derlet, ${ }^{4}$ Luca Anghinolfi, ${ }^{1,2,5}$ Ana Balan, ${ }^{3}$ Rajesh V. Chopdekar, ${ }^{1,3}$ \\ Marcus Wyss, ${ }^{1,6}$ Sebastian Gliga, ${ }^{1,2}$ Frithjof Nolting, ${ }^{3}$ and Laura J. Heyderman ${ }^{1,2, \dagger}$ \\ ${ }^{1}$ Laboratory for Micro- and Nanotechnology, Paul Scherrer Institute, Switzerland \\ ${ }^{2}$ Laboratory for Mesoscopic Systems, Department of Materials, ETH Zurich, 8093 Zurich, Switzerland \\ ${ }^{3}$ Swiss Light Source, Paul Scherrer Institute, 5232 Villigen PSI, Switzerland \\ ${ }^{4}$ Condensed Matter Theory Group, NUM, Paul Scherrer Institute, 5232 Villigen PSI, Switzerland \\ ${ }^{5}$ Laboratory for Neutron Scattering, Paul Scherrer Institute, 5232 Villigen PSI, Switzerland \\ ${ }^{6}$ Swiss Nanoscience Institute, University of Basel, Klingelbergstrasse 82, 4056 Basel, Switzerland \\ (Received 28 November 2013; revised manuscript received 14 May 2014; published 9 June 2014)
}

\begin{abstract}
We have performed a study of thermally driven magnetic relaxation in building blocks of artificial kagome spin ice. For room-temperature measurements, we observe that low-energy states are accessed with high efficiency, particularly in structures with strong dipolar coupling and with low thicknesses. With carefully tuned heating experiments, we demonstrate how thermally active artificial spin ice systems relax magnetically from higherenergy states and eventually fall into low-energy states. The methods applied in our work offer the possibility to observe the thermodynamics of artificial spin ice systems in real space and time, and provide a way to directly investigate the nature of complex stochastic processes.
\end{abstract}

DOI: 10.1103/PhysRevB.89.214405

PACS number(s): 75.75.Jn, 75.78.-n

\section{INTRODUCTION}

Frustration is a phenomenon that arises when competing interactions within a system can not be satisfied at the same time, leading to highly degenerate low-energy states. Systems incorporating frustration have long captured the interest of the scientific community due to their fascinating behavior including spin-freezing phenomena [1,2] and history dependent processes [3]. In addition, the understanding of their behavior leads to the interpretation of related complex systems. For example, spin-glass models, derived from extensive experimental research, have found their way into various other fields, providing a basis for modeling neural networks [4] and protein dynamics [5], and the generation of error-correcting codes [6].

Geometric magnetic frustration is a particular case where both the lattice geometry and the magnetic interaction are responsible for the frustration. A prominent example incorporating this kind of frustration is pyrochlore spin ice [7], which has drawn great interest, not only because of the associated thermodynamics that strongly resembles water ice $[8,9]$, but also due to excitations, which are referred to as emergent magnetic monopoles [10-13]. However, exploration of these pyrochlore systems relies on either measuring macroscopic quantities such as magnetic susceptibility [2] or using scattering techniques [12], which deliver reciprocal space information about the magnetic configuration.

Recently, much effort has been directed towards investigations of artificial spin ice [14-16], which is considered to be a two-dimensional analog of pyrochlore spin ice. This model system consists of dipolar-coupled monodomain nanomagnets arranged in two-dimensional geometries, such as artificial square ice [14,17,18] and artificial kagome spin ice [19-23]. In artificial square ice, dipolar coupling between the nanomagnets

\footnotetext{
*armin.kleibert@psi.ch

†laura.heyderman@psi.ch
}

will have a preferred moment configuration of two moments pointing into a vertex and two moments pointing out of a vertex where four nanomagnets meet, thus mimicking the two-in-two-out ice-rule spin alignment in pyrochlore spin ice [14]. In artificial kagome spin ice, a vertex is defined as the central point of each triplet of nanomagnets whose axial directions make an angle of $120^{\circ}$ with each other [24]. Here, the ice rule dictates a two-in-one-out or one-in-two-out vertex configuration. Such a kagome spin ice phase arises in pyrochlore spin ice when a magnetic field is applied along the [111] direction [25].

Artificial spin ice delivers the possibility to investigate frustration directly, observing the magnetic degrees of freedom using appropriate imaging methods such as magnetic force microscopy [14], Lorentz microscopy [20], or x-ray photoemission electron microscopy [21]. So far, most artificial spin ice systems were patterned from permalloy or cobalt films with thicknesses between 20 and $30 \mathrm{~nm}$. In this thickness range, the magnetic moments are static and the majority of experiments have focused on field driven processes, either consisting of demagnetizing protocols that involved rotating a sample in a decreasing magnetic field in order to achieve low-energy states [14,20,21] or magnetization reversal experiments with observations of emergent magnetic monopoles [22,23].

In order to achieve an improved analog to the bulk spin ice, one of the key goals in the field has been to realize and investigate thermally active artificial spin ice. Morgan et al. [17] observed that a long-range ordered state can be accessed in an as-grown artificial square ice, indicating that the ordering process occurs during the early stages of film deposition. This suggests the possibility to obtain a thermally active artificial spin ice in ultrathin films and, in recent work on patterned ultrathin Fe films [26], it was shown how remanent configurations of artificial square ice, which are frozen at low temperatures, started to "melt" with increasing temperature via thermally induced moment reorientations in the nanomagnets. It was also recently demonstrated that long-range ordered low-energy states can be achieved via 
(a)

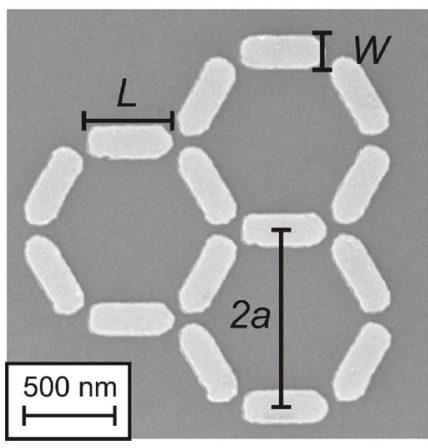

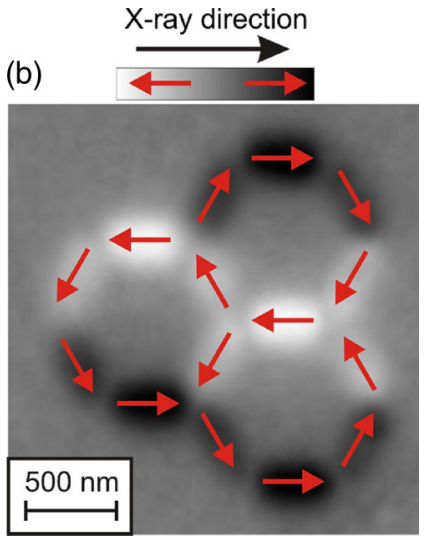

FIG. 1. (Color online) Three-ring building blocks of artificial kagome spin ice: (a) SEM image of a three-ring structure with nanomagnets of length $L=470 \mathrm{~nm}$, width $W=170 \mathrm{~nm}$, and thickness $d=5 \mathrm{~nm}$ patterned within a lattice parameter $a=500 \mathrm{~nm}$. (b) Corresponding magnetic contrast image from which the orientation of the nanomagnet moments can be determined.

thermal annealing $[18,27,28]$. Finally, time-resolved realspace observations revealed both thermally activated moment reorientations and magnetic ordering in building blocks of artificial kagome spin ice with up to three rings [29] and extended arrays of artificial square ice [18].

In the present work, we focus on the highly frustrated artificial kagome spin ice [see Fig. 1]. The magnetic configurations at a vertex obeying the ice-rule [24] involve either two moments pointing towards and one moment pointing out of the vertex where three nanomagnets meet (two-in/one-out) or vice versa (one-in/two-out), as shown in Fig. 2(a). In particular, we investigate the possibility to achieve low-energy states in the building block structures [21,29] via thermal annealing. Using a bottom-up approach [30-32], our work covers systems ranging from one-ring structures up to sevenring structures [see Figs. 1 and 2(b)-2(f)]. Compared to extended arrays, these building block structures possess the advantage that their energy spectra can be easily calculated and their magnetic states can be classified into different energy bands [21].

We have performed film-thickness-dependent measurements at room temperature on the artificial kagome spin ice structures, which were fabricated by patterning a permalloy (Ni80\%Fe20\%) wedge film [see Fig. 3(a)]. This approach allows us to vary the energy barriers for thermal fluctuations, so that quasistatic states are achieved at higher thicknesses, while superparamagnetic fluctuations occur at lower thicknesses. Employing x-ray magnetic circular dichroism (XMCD) [33] in a photoemission electron microscope (PEEM) [34], we are then able to directly observe the magnetic states and classify them energetically as a function of time.

We begin with the experimental details, including sample fabrication and magnetic imaging as well as an outline of our simulation strategy in Sec. II. We then report on how the number of accessed low-energy states changes with film thickness and dipolar coupling, and demonstrate the possibility to achieve artificial spin ice structures with fluctuating

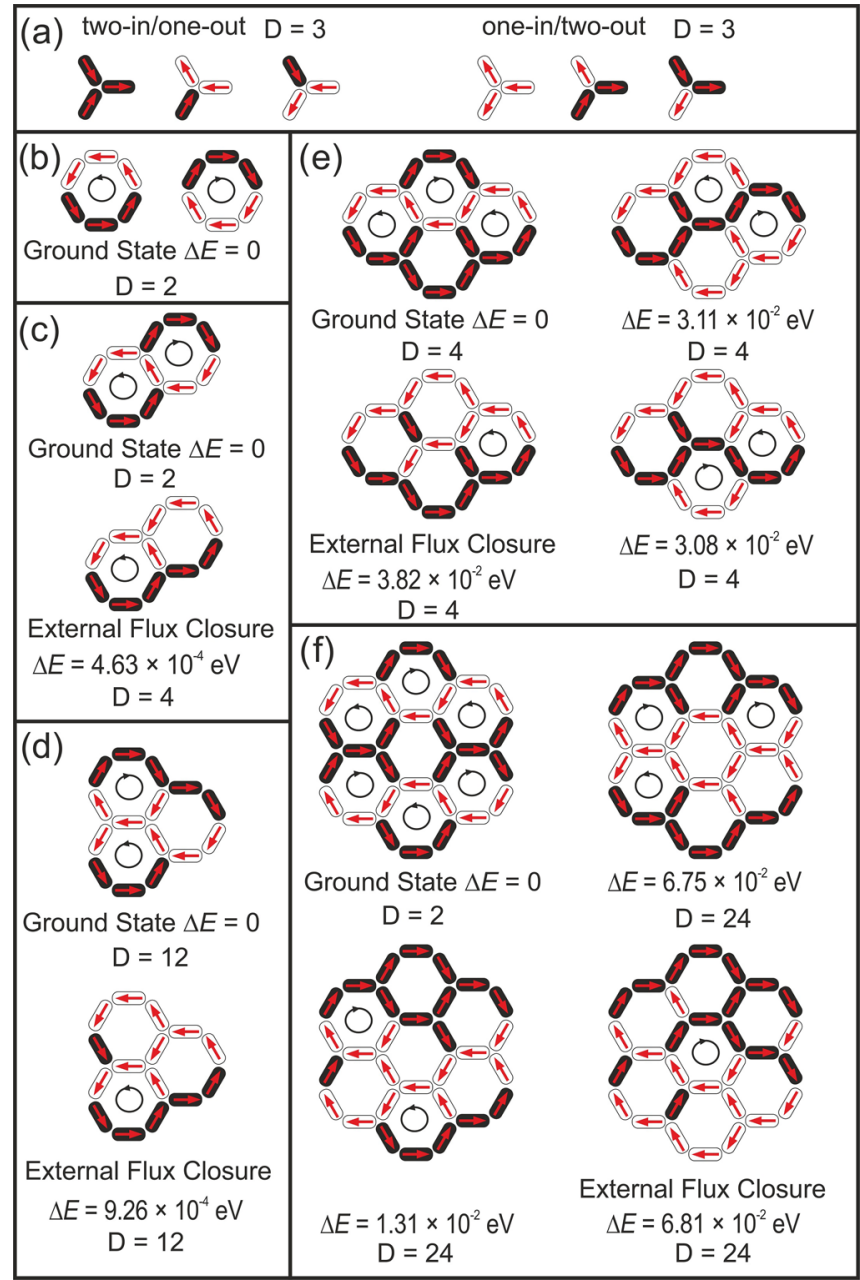

FIG. 2. (Color online) (a) The six degenerate ice-rule moment configurations at a vertex of artificial kagome spin ice. (b)-(f) Schematics of building block structures of artificial kagome spin ice and some of their low-energy states shown together with their degeneracy, $D$, and energy, $\Delta E$, relative to the ground-state energy for a lattice parameter $a=500 \mathrm{~nm}$. While all shown states belong to the first (lowest-energy) band, all structures possess a ground state, as indicated, and with an increasing system size there is an increasing number of degenerate low-energy states belonging to this band.

moments at room temperature in Sec. III. In Sec. IV, we present direct observations of thermal relaxation processes in the kagome building block structures, and finally, in Sec. V, we demonstrate for larger ring numbers how the system explores a number of low-energy states (moment configurations that belong to the first energy band) via thermally induced moment reorientations and reveal an increasing difficulty to access the ground state with increasing system size.

\section{METHODS}

\section{A. Experimental}

The samples were fabricated by electron beam lithography using a Vistec EBPG 5000 Plus electron-beam writer. The patterns were exposed on a silicon (100) substrate, spincoated with a 70 -nm-thick layer of polymethylmethacrylate 
(a)

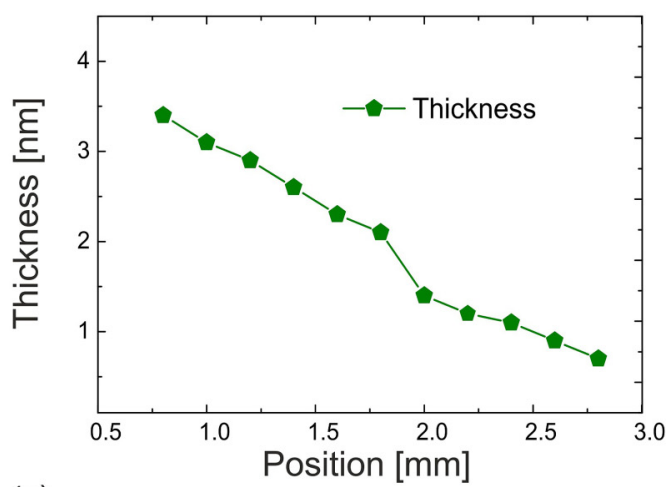

(c)

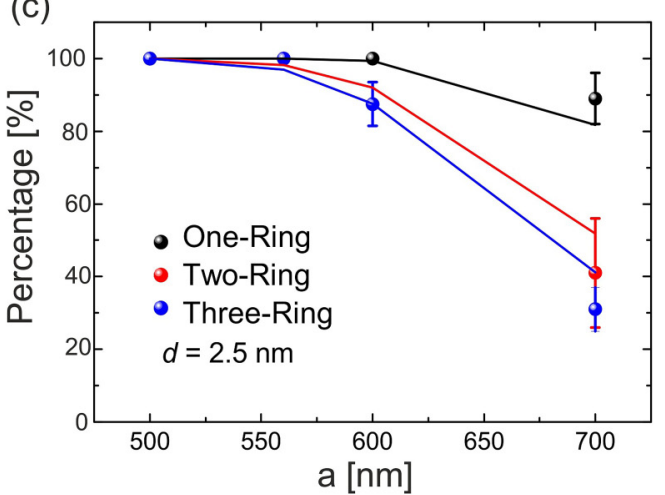

(b)

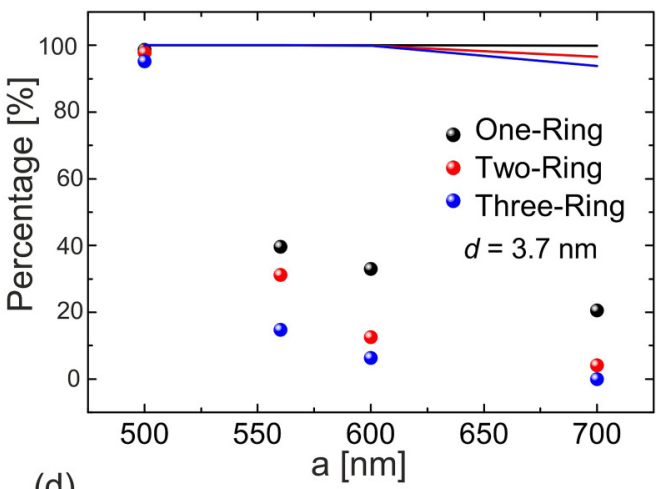

(d)

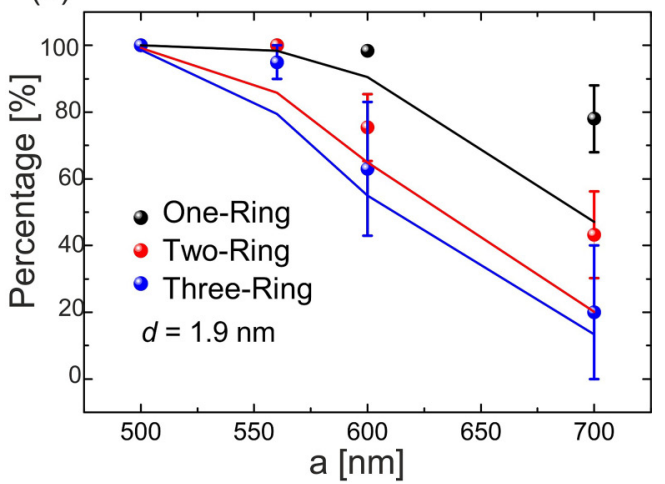

FIG. 3. (Color online) (a) Thickness of the permalloy film measured at different positions across a wedge sample. (b)-(d) Percentages of low-energy states observed at different thicknesses $d$ as a function of the lattice parameter $a$ at $T=300 \mathrm{~K}$ (filled circles). At lower film thicknesses in (c) and (d), we observe spontaneous configurational changes within the time scale of XMCD imaging. Recording image sequences, consisting of 10 images per sequence, we determine the average percentages of low-energy states achieved with the standard deviations given as error bars. Colored lines correspond to thermal occupancies of low-energy states for one-, two- and three-ring structures derived from Eq. (2). The filled circles are the PEEM data and both the experimental measurements as well as the simulations were performed for lattice parameters $a=500,560,600$, and $700 \mathrm{~nm}$.

(PMMA) resist. A ferromagnetic permalloy wedge film was then created by moving the sample manually beneath a fixed shutter during film deposition (base pressure: $6 \times 10^{-6}$ mbar, evaporation pressure: $6 \times 10^{-5}$ mbar), with a thickness $d$ that ranges from 0.7 to $3.7 \mathrm{~nm}$ over a distance of approximately $2 \mathrm{~mm}$ [see Fig. 3(a)]. Finally, a 2-nm-thick aluminum capping layer was added to protect the structures from oxidation. Lift-off in acetone was then used to remove the remaining resist and all unwanted magnetic material. The resulting patterned nanomagnets have lengths of $470 \mathrm{~nm}$ and widths of $170 \mathrm{~nm}$, and were arranged on a kagome lattice with lattice parameter $a$ [see Fig. 1(a)], ranging between 500 and $800 \mathrm{~nm}$. The variation of the film thickness across the sample was measured with an atomic force microscope (AFM) [Fig. 3(a)].

Magnetic contrast images are obtained in a PEEM by pixelwise division of images recorded at the $\mathrm{Fe} L_{3}$ edge with circularly right and left polarized x-rays. The resulting $\mathrm{XMCD}$ contrast is a measure of the projection of the magnetic moments onto the $\mathrm{x}$-ray propagation vector. As a result, magnetic moments pointing towards the $\mathrm{x}$-ray direction appear dark while moments pointing in the opposite direction appear bright [see Fig. 1(b)]. For each polarization, an exposure time of one second is chosen and repeated three times for averaging. Switching polarizations usually takes two seconds, giving an overall time for obtaining an XMCD image of roughly eight seconds. Accordingly, only magnetic configurations with a residence time longer than this can be reliably determined from the XMCD images. For all experiments, we measured 75 one-ring, 48 two-ring, 48 three-ring, 48 fourring, and 36 seven-ring structures, all patterned on the same substrate.

The applied annealing protocol can be broken down into two major steps. The first step is performed below the nanomagnet blocking temperature and involves the application of a magnetic field $(35 \mathrm{mT})$, so that all moments are set to point towards the direction of the applied field, which points parallel to the long axis of one set of nanomagnets. Thus, the magnetic configurations are initially in well-defined remanent high-energy states. As a second step, the sample is heated slightly above the blocking temperature and spatially resolved real-time observations of thermal relaxation of the artificial kagome spin ice structures are carried out. In order to avoid possible sample damage that can occur as a result of strong heating, the experiments are performed close to room temperature. The ideal film thickness then has to be determined for which the moments are static at room temperature and some heating (up to $320-330 \mathrm{~K}$ ) is required in order to observe configurational changes within the temporal resolution of XMCD imaging. 


\section{B. Simulation}

In determining the energy of a particular magnetic configuration, two fundamental interactions are known to contribute to the total magnetic energy; the exchange and the magnetostatic interaction. For the very thin nanomagnets considered here (where the thickness of each nanomagnet is never more than a few nanometres), the exchange contribution dominates the internal relaxation, and all internal moments can be considered to be perfectly aligned. This considerably simplifies the calculation of the total magnetic energy, since the intrananomagnet magnetostatic energy (shape anisotropy) is now minimized when the total moment of each nanomagnet is aligned either parallel or antiparallel along its axial length. The only remaining contribution is the internanomagnet magnetostatic energy, which is now the sole contribution to the energy. This term can be evaluated in a number of ways, one of which is to discretize each nanomagnet into an array of sufficiently small cells such that the magnetization is uniform across the cell. Doing so allows the magnetostatic energy $\left(E_{\mathrm{id}}\right)$ to be represented as a double summation over cells, which interact via the dipolar interaction:

$$
\begin{aligned}
E_{\mathrm{id}}= & \frac{\mu_{0}\left(\Delta V M_{\mathrm{s}}\right)^{2}}{8 \pi} \sum_{i, j} \frac{1}{\left|\mathbf{r}_{i j}\right|^{3}}\left[\hat{\mathbf{m}}_{i} \cdot \hat{\mathbf{m}}_{j}\right. \\
& \left.-\frac{3}{\left|\mathbf{r}_{i j}\right|^{2}}\left(\hat{\mathbf{m}}_{i} \cdot \mathbf{r}_{i j}\right)\left(\hat{\mathbf{m}}_{j} \cdot \mathbf{r}_{i j}\right)\right] .
\end{aligned}
$$

Here, $\hat{\mathbf{m}}_{i}$ is the moment direction within the $i$ th cell and $\mathbf{r}_{i j}$ is the distance vector between cells $i$ and $j$, which may either be in the same or different nanomagnets within a given kagome structure. $\Delta V$ is the volume of each cell and $M_{\mathrm{s}}$ is the nanomagnet's magnetization. Thus the dipolar energy scale is set by the constant $\left(\Delta V M_{\mathrm{s}}\right)^{2}$, where $\Delta V M_{\mathrm{s}}$ is the total moment of each cell.

While the above procedure is able to accurately reproduce the correct energetics for a given choice of $M_{\mathrm{s}}$ and sufficiently small cell size, it rapidly becomes computationally intensive for increasing kagome system size when all of the possible magnetic configurations are enumerated. Indeed, for an $N$ nanomagnet kagome system, the number of possible magnetic configurations equals $2^{N}$, indicating exponential growth with increasing system size. A compromise between accuracy and simplicity is therefore needed and our past work has shown that the physically relevant energetics of the kagome (and square lattice) energy spectrum can be well reproduced by treating each nanomagnet as either a point dipole source [21] or an infinitesimally thin compass needle $[18,29]$ that is discretized into a linear chain of cells. In the present work, the latter approximation is taken. Indeed, explicitly considering the width of the nanomagnet was found to have little effect on the final energy spectrum. Such an approach may be seen as a simplification to the more traditional micromagnetic calculations, and is equivalent to the dipolar model considered by Möller and Moessner [37].

For the present work, each nanomagnet was represented as a linear chain of nine cells, each with a magnetic moment equal to $V M_{\mathrm{s}} / 9$, where $V$ is the total volume of each nanomagnet, taking the nanomagnet dimensions as in the experiment (length $L=470 \mathrm{~nm}$, width $W=170 \mathrm{~nm}$ and thickness $d=1.9-3.7 \mathrm{~nm})$. To obtain the best overall agreement with experiment, a magnetization of $3.75 \times 10^{5} \mathrm{~A} / \mathrm{m}$ was required, which is a value below that for bulk permalloy $\left(8 \times 10^{5} \mathrm{~A} / \mathrm{m}\right)$, but in agreement with values measured by magnetometry for ultrathin patterned and unpatterned permalloy films [18,29]. The resulting energies of the finite kagome systems relative to the lowest-energy state using this model are shown in Figs. 2(b)-2(f).

More generally, the simulations reveal a density of states for each finite kagome system that is organized into bands separated by energy gaps [see upper inset in Fig. 5], with the low-energy states occupying the first (lowest) band. For one-, two- and three-ring structures the first band consists of 2, 6, and 24 low-energy states, respectively [21,29]. Within the first band, each finite system will have a particular set of absolute lowest energy states (ground states) [see Figs. 2(b)-2(f)]. These ground-state configurations are characterized by a maximization in the number of rings in the vortex state $[21,35,36]$, i.e., all nanomagnet moments pointing in same sense around a ring, either clockwise or anticlockwise [21,35,36], which corresponds to the smallest closed loop. Besides these ground states, there is a certain number of additional low-energy states in the first band. For two- and three-ring structures, the so-called external flux closure states [21], where perimeter nanomagnets have all of their moments pointing head to tail [Figs. 2(b)-2(f)], are the only other low-energy configurations. For structures with four rings or more, further types of configurations belong to the first band [see Figs. 2(e) and 2(f)]. In the second band, the three-nanomagnet (frustrated) vertices still obey the ice rule, but there are a number of head-tohead and tail-to-tail moment alignments appearing along the perimeter at two-nanomagnet (unfrustrated) vertices. For the case of one-, two-, and three-ring structures, the second band consists of 24,32 , and 179 configurations, respectively [21].

\section{THICKNESS-DEPENDENT ORDERING IN FINITE KAGOME RING STRUCTURES}

Previously, it was found that, on application of demagnetizing field protocols [21], there is a significant decrease in the ability to achieve the low-energy states in the first band with increasing system size. Here, we investigate the percentage of low-energy states resulting from thermal activation of magnetic fluctuations, studying their occupancy at room temperature following deposition without applying external fields and without heating.

In these as-grown samples, we find a strong thickness dependence of the percentage of low-energy states achieved in arrays of one-, two- and three-ring structures [see Figs. 3(b)-3(d)]. At a thickness of $d=3.7 \mathrm{~nm}$ [see Fig. 3(b)], only the strongest coupling $(a=500 \mathrm{~nm})$ results in $100 \%$ low-energy states but, at lower film thicknesses, higher percentages of low-energy states are achieved at all lattice parameters [see Figs. 3(c) and 3(d)]. For all structure types and all film thicknesses, reducing the dipolar coupling (going from $a=500 \mathrm{~nm}$ to $a=700 \mathrm{~nm}$ ) significantly decreases the energy gap between the first and the higher-energy bands. This results in a more probable occupation of excited states so that more states in higher energy bands are accessed. While the moment configurations are static at $d=3.7 \mathrm{~nm}$, 
we start to see spontaneous configurational changes within the time scale of XMCD imaging (7-9 seconds) at lower film thicknesses (2.5 and $1.9 \mathrm{~nm}$ ) [see Figs. 3(c) and 3(d)]. Here we record image sequences consisting of 10 images per sequence (80-90 seconds of observation), and determine the average percentages of low-energy states achieved with the standard deviations as shown in Figs. 3(c) and 3(d).

The strong dependence on film thickness of the experimental percentages of low-energy states [see Figs. 3(b)-3(d)] suggests a strong connection between film thickness and the time scale of the magnetic relaxation. This may be rationalized by assuming that the relaxation time scale of a nanomagnet moment will follow an Arrhenius-type behavior [18,29] whose activation energy will be some increasing function of the spatial extent of the nanomagnets. Because of this, small changes in film thickness are expected to lead to very significant changes in relaxation time scales and therefore the percentages of low-energy states as seen in Figs. 3(b)-3(d). To gain quantitative insight into the results of Fig. 3, we consider the scenario where the relaxation time scale is well within the experimental time-scale, thus allowing the system to achieve thermal equilibrium. In this regime, equilibrium statistical mechanics can be used to calculate the average thermal occupancy of the lowest-energy band:

$$
\langle O\rangle=\frac{\sum_{n \in \text { first band states }} \exp \left(-\frac{\Delta E_{n}}{k_{\mathrm{b}} T}\right)}{\sum_{n \in \text { all states }} \exp \left(-\frac{\Delta E_{n}}{k_{\mathrm{b}} T}\right)},
$$

where $n$ indexes each magnetic configuration and $\Delta E_{n}=$ $E_{n}-E_{0}$ in which $E_{0}$ is the lowest-energy magnetic configuration and $E_{n}$ is the energy of the $n$th configuration obtained from the modeling method described in Sec. II B.

The corresponding calculated occupancies for the three considered nanomagnet thicknesses are displayed as lines in Figs. 3(b)-3(d). While reasonable agreement is seen for the 2.5-nm and 1.9-nm nanomagnet thicknesses, there is very poor agreement for the kagome systems with nanomagnet thickness of $3.7 \mathrm{~nm}$. This result may be understood by the fact that for the 3.7-nm-thick structures, the relaxation time is expected to be much larger than the time scale over which the experiment was performed, and thus the observed as-grown states are generally out of equilibrium at $300 \mathrm{~K}$ for all but the smallest lattice constant. Indeed, the very rapid drop in occupancy with increasing lattice constant seen in Fig. 3(b) reflects that with decreasing dipolar coupling, the kagome building block structures find it more and more difficult to distinguish the particular pathways leading to the lowest energy states [29]. Thus the statistics seen in Fig. 3(b) originate from quenched disorder and therefore are very dependent on the thermal history and sample production method. In contrast, for the nanomagnets with a thickness of 1.9 and $2.5 \mathrm{~nm}$ [Figs. 3(c) and 3(d)], the magnetization dynamics are within the experimental time scale, and the system has time to fully relax, resulting in annealed (thermal) disorder and hence agreement with the predictions of Eq. (2).

For all the samples, we have assumed that the magnetic volume is equivalent to the geometrical volume of the nanomagnets and the saturation magnetization is equal to $3.75 \times 10^{5} \mathrm{~A} / \mathrm{m}$. However, the poorer agreement between the experiment and simulation seen for the thinnest sample
[Fig. 3(d), when compared to Fig. 3(c)] may indicate that, in this regime of film thickness, changes in the permalloy microstructure could lead to both a modification of $M_{\mathrm{s}}$ and to a magnetic volume $V$ that is not a simple linear function of film thickness. This would alter the dipolar energy scale that is controlled by $V M_{\mathrm{s}}$, which in turn changes the energy spectrum of magnetic configurations and the associated thermal occupancies.

\section{REAL-TIME OBSERVATIONS OF THERMALLY-DRIVEN RELAXATION}

In order to visualize the thermal relaxation processes in real time, structures were chosen such that their film thickness resulted in static moments at room temperature, only requiring heating to $320-330 \mathrm{~K}$ in order to reach temperatures slightly above the blocking temperature and thus observe configurational changes within the temporal resolution of XMCD imaging. Here, a film thickness of approximately $3 \mathrm{~nm}$ was found to be ideal for observing moment reorientations of the nanomagnets.

Following the annealing protocol described in Sec. II, we first apply a saturating magnetic field parallel to the long axis of a set of nanomagnets, and therefore, on removing the field, create moment configurations of one-, two-, three-, and fourring structures that belong to the second energy band. Initially, the energy barrier is high enough to prevent a spontaneous relaxation at room temperature within the time scale of a single measurement. By heating up to $330 \mathrm{~K}$, we can observe how kagome building block structures relax towards the first energy band via thermally activated moment reorientations.

This is demonstrated for a strongly-coupled three-ring structure $(a=500 \mathrm{~nm})$ in Figs. 4(a)-4(h). Following the application of a saturating magnetic field to the left, the configuration is set to an energetically higher state, which belongs to the second energy band with all moments pointing towards the left. With the onset of thermally induced moment reorientations on heating, we observe spontaneous configurational changes between the high-energy states with similar energies within the second band [see Figs. 4(a)-4(e)] during approximately two minutes. Eventually, the reversal of the inner horizontal nanomagnet moment, together with two nearest-neighbor reversals, leads to the formation of two rings in clockwise and anticlockwise vortex states, and a reduced dipolar energy [see Figs. 4(f)-4(g)]. From this state, the system is only one moment flip away from the first energy band and roughly three minutes after the start of the experiment, the first band is reached [see Fig. 4(h)]. As soon as the moment configurations fall into their low-energy valley (first energy band), thermal excitations enable them to freely wander between the states, which belong to this band [29] and, with an energy gap of approximately $0.4 \mathrm{eV}$ to the second band [see upper inset in Fig. 5], the system will spend very little time in configurations belonging to the second band [29].

Applying the same method of saturating and heating of one-, two-, and four-ring structures revealed similar relaxation processes, always starting with the higher energy states and relaxing to the low-energy states in the first band. In order to determine the percentages of low-energy states after relaxation (after approximately one hour), we cool the system down 


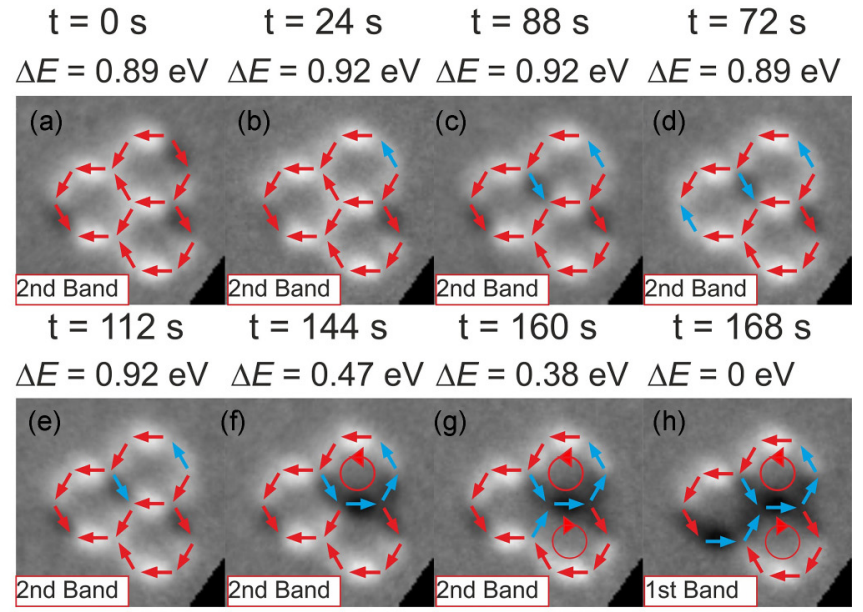

(i)

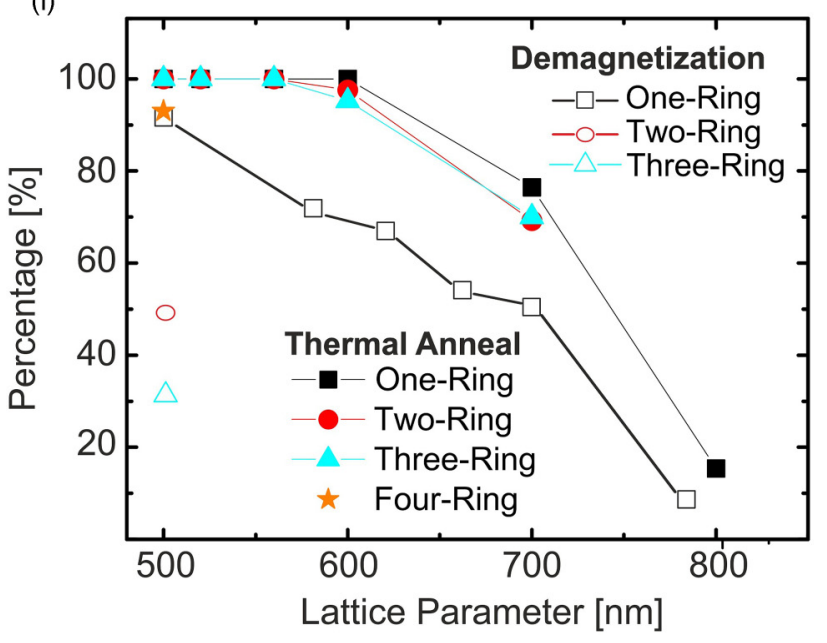

FIG. 4. (Color online) (a)-(h) Thermal relaxation of a three-ring structure ( $a=500 \mathrm{~nm}$ and $T=330 \mathrm{~K}$ ), going from an energetically higher state to one of 24 degenerate low-energy states within 168 seconds. Blue arrows in (a) to (h) represent reversed nanomagnet moments compared to the initial moment configuration in (a). The time $t$ and the energy relative to the ground state $\Delta E$ are shown to visualize the evolution of the system within the energy landscape. (i) Percentages of low-energy states achieved (closed shapes) in arrays of kagome building blocks after thermal annealing involving heating up to $330 \mathrm{~K}$ and cooling down to $300 \mathrm{~K}$ following relaxation. While the number of rings (up to three) does not seem to have a large effect on the numbers of low-energy states achieved, an increasing lattice parameter (decreasing dipolar coupling) significantly affects the occupancy of the lowest energy states, which can be explained by a strong decrease in the energy gap between the first and the second energy bands with decreasing dipolar coupling. The percentages of low-energy states achieved following a demagnetizing protocol [21] are given for comparison (open squares, circles and triangles).

to room temperature, so that the observed low-energy states are frozen and can be counted. Up to a lattice parameter $a=600 \mathrm{~nm}$, we see 90\%-100\% low-energy states achieved for all kagome building block structures [see Fig. 4(i)]. This demonstrates the high efficiency of thermal annealing for obtaining low-energy states compared to field-driven demagnetizing procedures, where an increasing system size

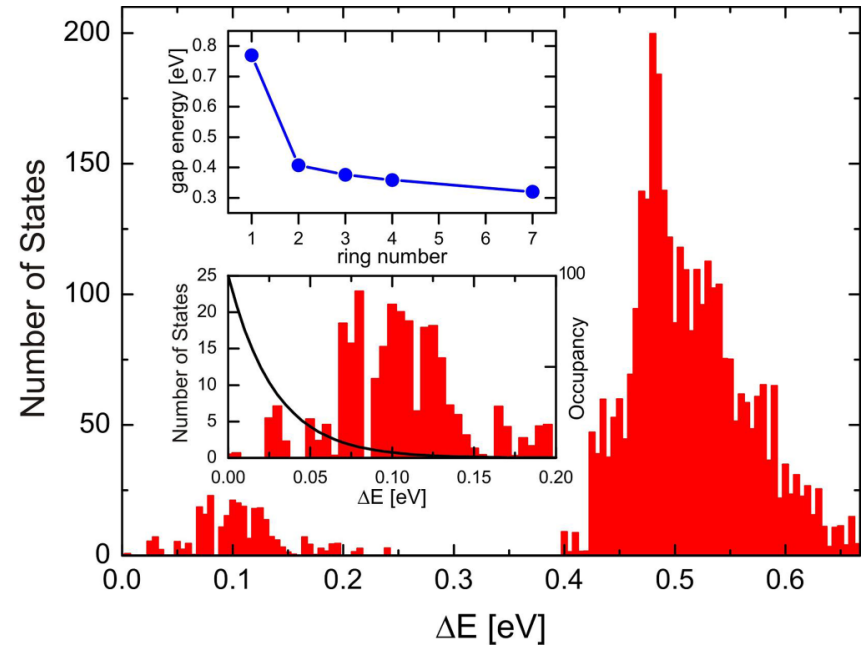

FIG. 5. (Color online) Density of states calculated for a sevenring kagome building block structure $(a=500 \mathrm{~nm})$ revealing a gap between the first and the second energy bands. The upper inset demonstrates the systematic decrease of the energy gap with increasing system size. The lower inset focuses on the first (lowest) energy band, with the black line being a plot of the Boltzmann factor at a temperature of $330 \mathrm{~K}$. Multiplying the Boltzmann factor (black line) by the calculated density of states gives the predicted thermal occupancy at $T=330 \mathrm{~K}$.

leads to a significant decrease in the percentage of low-energy states achieved [21]. The percentages of low-energy states achieved following field demagnetization [21] are shown in Fig. 4(i) for comparison. With increasing lattice parameter (decreasing dipolar coupling), there is a decrease in the percentage of low-energy states from 90\%-100\% at $a=$ $600 \mathrm{~nm}$ to only $16 \%$ for single-ring structures at $a=800 \mathrm{~nm}$ [see Fig. 4(i)], demonstrating the important role of dipolar coupling between the nanomagnets for achieving the low-energy states.

\section{EFFECT OF SYSTEM SIZE ON MAGNETIZATION DYNAMICS}

Artificial kagome spin ice is a highly frustrated system that has a strongly increasing density of low-energy states with increasing system size [21,29]. For the case of a onering structure, the first energy band consists of a doubly degenerate low-energy vortex state [21,29], whereas for a four-ring structure, it consists of 104 low-energy states. For even larger finite systems, this number rapidly grows and when simulating a seven-ring system, direct enumeration of the states becomes intractable and more approximate methods are needed. Here, Monte Carlo methods are used to statistically sample the low-energy states obtained from the modeling approach outlined in Sec. II B. This simulation was performed at $T=1000 \mathrm{~K}$, a temperature which is sufficient to accurately probe the full energy spectrum of the first band plus much of the second energy band. The final density of states is obtained by dividing the energy spectrum obtained from the Monte Carlo method by the Boltzmann factor $\exp \left[\Delta E /\left(k_{\mathrm{b}} T\right)\right]$ with $T=1000 \mathrm{~K}$. The density of states for the seven-ring artificial kagome spin ice structure, in which the zero energy is taken 
to be the energy of the lowest energy state, is displayed in Fig. 5, with the two energy bands separated by an energy gap. In the upper inset in Fig. 5, this gap, defined as the difference between the lowest energy value of the second band and the average energy of the lowest energy states in the first band, is plotted as a function of the kagome ring number. One sees that as the structure size increases, the gap decreases and will converge to a value for very large systems that corresponds to the energy associated with locally breaking the ice rule.

For experiments at all system sizes performed at $330 \mathrm{~K}$, configurations that break the ice rule or constitute a high energy excitation of the perimeter nanomagnets are never observed. For the case of the seven-ring structure, this can be understood via the lower inset in Fig. 5, which focuses on the first energy band and plots the Boltzmann factor at $330 \mathrm{~K}$ (black line), indicating the mean thermal occupancy at this temperature (energy spectrum $=$ density of states $\times$ Boltzmann factor), and demonstrating that excitations outside the first energy band are unlikely. This also shows that it is unlikely that the doubly degenerate ground state [see Fig. 2(f)] will be observed with the most likely states occurring at $\sim k_{\mathrm{b}} T=k_{\mathrm{b}} \times 330 \mathrm{~K}=0.03 \mathrm{eV}$.

By determining the percentages of ground states achieved for all strongly coupled $(\mathrm{a}=500 \mathrm{~nm})$ structures following the annealing protocol described in Sec. II, we confirm the increased difficulty in accessing one of the ground states. For one-, two-, three-, and four-ring structures, the percentages of ground states observed are $100 \%, 32 \%, 62 \%$, and $13 \%$, respectively. This is in line with the expected percentages of $100 \%, 33 \%, 50 \%$, and $4 \%$ for the same structures, taking into account the degeneracy of low-energy states given in Figs. 2(b)-2(f). For the seven-ring structure, while there is thermal relaxation from the magnetic-field-defined high-energy state to the low-energy states belonging to the first energy band, we did not observe the ground state, despite repeating the annealing procedure on several seven-ring structures and observing up to 100 different low-energy states over a period of an hour. It therefore appears that lower temperatures over an extended period of time would be needed to observe the ground state in the seven-ring structure. Unfortunately, though, these temperatures are likely to be below the system's blocking temperature, resulting in a shift from probing equilibrium states (annealed disorder) to probing out-of-equilibrium states (quenched disorder).

An example of the thermal relaxation in the seven-ring structure is given in Fig. 6. Initially, after applying a saturating magnetic field, all moments point towards the right. Starting from this remanent state, the moments of the horizontal inner nanomagnets are unlikely to switch, as this would result in ice-rule violations. Therefore nonhorizontal nanomagnets reverse their moments first and initiate subsequent moment reversals while strictly maintaining the ice rule. This leads to the creation of rings with clockwise and anticlockwise vortex states [Figs. 6(a)-6(c)] or strings of reversed moments [Figs. 6(h)-6(j)]. In addition to vortex states, moment reversal of sequential perimeter nanomagnets leads to a low-energy external flux closure state [see Figs. 6(d)-6(h)]. Once this state is achieved, further configurational changes are dominated by moment reversals of inner nanomagnets, all locally obeying the ice rule [see Figs. 6(i)-6(1)]. This continued exploration of
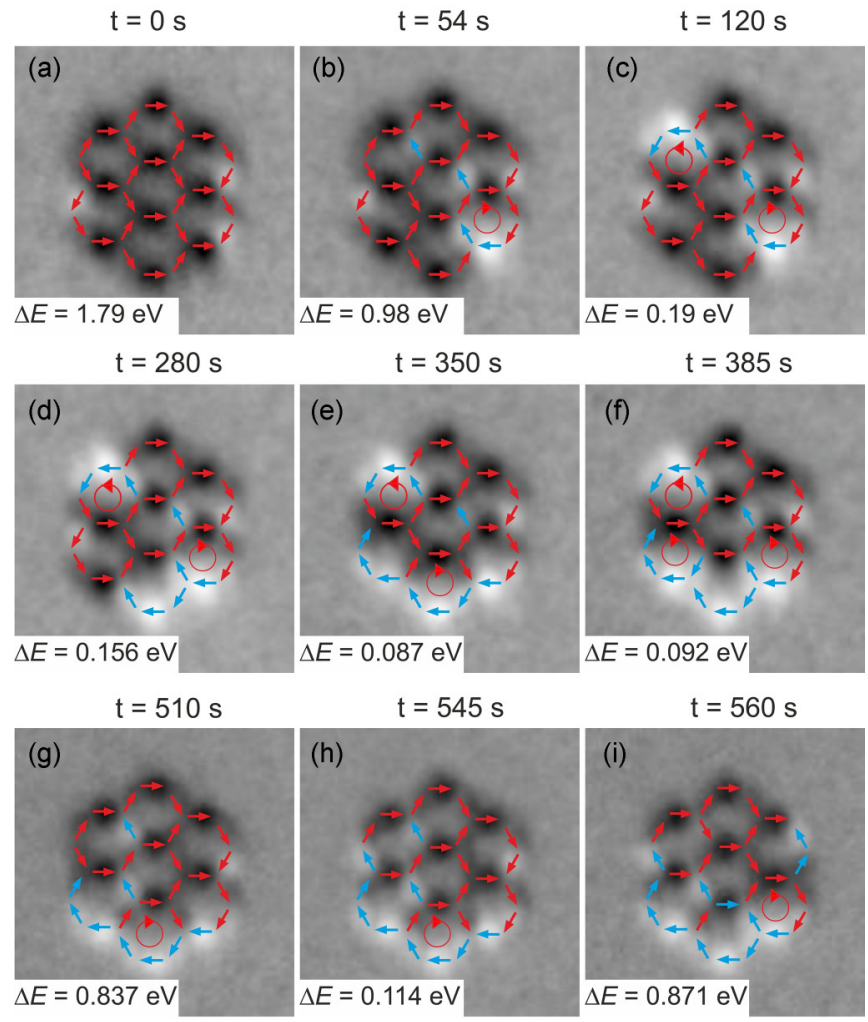

$t=575 s$

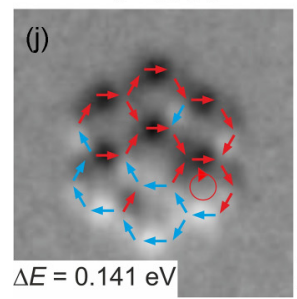

$t=605 s$

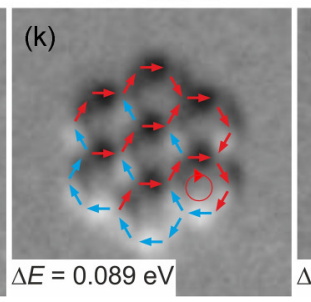

$t=645 s$

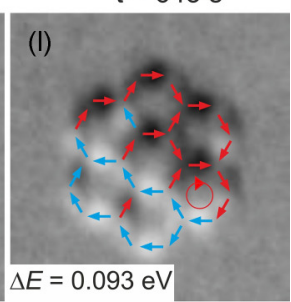

FIG. 6. (Color online) (a)-(h) Thermal relaxation of a seven-ring artificial kagome spin ice structure $(a=500 \mathrm{~nm})$, from a high-energy state to an external flux closure state (low-energy state), where perimeter nanomagnets have all their moments aligned head to tail. This is followed by a number of moment reorientations mainly in interior nanomagnets, leading to transitions between several lowenergy states of similar energies $\Delta E$ relative to the ground state (i)-(l). The ground state was never observed. Blue arrows in (b)-(l) represent reversed moments of nanomagnets, compared to the initial configuration in (a).

the low-energy sector of the energy landscape has also been reported for kagome building block structures of up to three rings [29].

\section{CONCLUDING REMARKS}

We have investigated the possibility of achieving lowenergy states in building blocks of artificial kagome spin ice in their as-grown state at different film thicknesses, without the application of an external field or additional heating above room temperature, and find that strong dipolar coupling is essential for achieving $100 \%$ low-energy states. In addition, we performed thermal relaxation experiments, observing how the structures evolve from a high-energy remanent state following 
saturation to the lowest energy states for system sizes of up to seven rings. In all cases, the final states following relaxation belonged to the first energy band, which are separated by a gap from the second band. For structures with one to four rings, we observed the ground-state configurations that occur within the spectrum of low-energy states. However, for the seven-ring structure, thermal fluctuations allowed the system to explore the large number of low-energy states without attaining the absolute lowest energy that corresponds to the long range order thought to exist in extended systems [37]. This is a direct result of the high frustration and extensive energy landscape encompassing a large number of degenerate low-energy states, and is in contrast to artificial square ice, which has a distinct gap between the ground state and the next energy band, leading to a perfect order following thermal relaxation [18].

While we demonstrate the high-efficiency of thermal annealing in the building blocks of artificial kagome spin ice, which is not seen when using demagnetizing protocols [21], on extrapolating our results to extended arrays of artificial kagome spin ice, we conclude that it will be difficult to achieve the ground state $[37,38]$ using the presently applied thermal annealing protocol. This could have different reasons. It may, for example, be that long range ordering is not able to set in at the temperatures used in our experiments. Therefore future work might move towards artificial kagome spin ice structures with much lower nanomagnet blocking temperatures, so that larger temperature ranges can be explored and the predicted phase transitions to long-range ordered ground-state configurations [37,38] can be accessed. It is also noted that the present work does not consider intrinsic disorder that, if sufficiently strong, would influence the dynamics [18] and may be partly responsible for the lack of observation of the lowest-energy configurations.
Other recently presented annealing procedures are based on heating above the Curie temperature and observing the frozen-in configurations with magnetic force microscopy after cooling back to room temperature $[27,28]$. In comparison, our combination of a patterned permalloy wedge and imaging with PEEM [18,29] has the main advantage that configurational changes can be spatially resolved as a function of time. The method presented here not only delivers the possibility for direct comparison with theoretical predictions of thermal behavior [37-42] but also the exciting prospect to directly observe and investigate the thermally driven behavior of emergent magnetic monopoles in artificial kagome spin ice. In contrast to field-driven experiments [22,23], where the magnetic monopole motion is determined by the orientation of the applied magnetic field, the thermally driven behavior of emergent magnetic monopoles will be unconstrained. Future observations of this kind will provide the possibility to draw direct comparisons with the low-temperature thermodynamics of pyrochlore spin ice [11-13].

\section{ACKNOWLEDGMENTS}

The authors would like to thank R. Schelldorfer, A. Weber, E. Deckardt, and V. Guzenko for their support. This work was supported by the Swiss National Science Foundation and the Swiss Nanoscience Institute, Basel, Switzerland. In addition, the research leading to these results has received funding from the European Community's Seventh Framework Programme (FP7/2007-2013) under Grant Agreement No. 290605 (COFUND: PSI-FELLOW). Part of this work was performed at the Surface/Interface:Microscopy (SIM) beam line of the Swiss Light Source, Paul Scherrer Institut, Villigen, Switzerland.
[1] W. Kinzel and K. H. Fischer, Solid State Commun. 23, 687 (1977).

[2] J. Snyder, J. S. Slusky, R. J. Cava, and P. Schiffer, Nature (London) 413, 48 (2001).

[3] Z. D. Chen, Phys. Rev. B 53, 6499 (1996).

[4] G. Toulouse, S. Dehaene, and J. P. Changeux, Proc. Natl. Acad. Sci. U.S.A. 83, 1695 (1986).

[5] J. D. Bryngelson and P. G. Wolynes, Proc. Natl. Acad. Sci. U.S.A. 84, 7524 (1987).

[6] N. Sourlas, Nature (London) 339, 693 (1989).

[7] M. J. Harris, S. T. Bramwell, D. F. McMorrow, T. Zeiske, and K. W. Godfrey, Phys. Rev. Lett. 79, 2554 (1997).

[8] L. Pauling, J. Am. Chem. Soc. 57, 2680 (1935).

[9] A. P. Ramirez, A. Hayashi, R. J. Cava, R. Siddharthan, and B. S. Shastry, Nature (London) 399, 333 (1999).

[10] C. Castelnovo, R. Moessner, and S. L. Sondhi, Nature (London) 451, 42 (2008).

[11] S. T. Bramwell, S. R. Giblin, S. Calder, R. Aldus, D. Prabhakaran, and T. Fennell, Nature (London) 461, 956 (2009).
[12] D. J. P. Morris, D. A. Tennant, S. A. Grigera, B. Klemke, C. Castelnovo, R. Moessner, C. Czternasty, M. Meissner, K. C. Rule, J.-U. Hoffmann, K. Kiefer, S. Gerischer, D. Slobinsky, and R. S. Perry, Science 326, 411 (2009).

[13] L. D. C. Jaubert and P. C. W. Holdsworth, Nat. Phys. 5, 258 (2009).

[14] R. F. Wang, C. Nisoli, R. S. Freitas, J. Li, W. McConville, B. J. Cooley, M. S. Lund, N. Samarth, C. Leighton, V. H. Crespi, and P. Schiffer, Nature (London) 439, 303 (2006).

[15] L. J. Heyderman and R. L. Stamps, J. Phys.: Conden. Matter 25, 363201 (2013).

[16] C. Nisoli, R. Moessner, and P. Schiffer, Rev. Mod. Phys. 85, 1473 (2013).

[17] J. P. Morgan, A. Stein, S. Langridge, and C. H. Marrows, Nat. Phys. 7, 75 (2011).

[18] A. Farhan, P. M. Derlet, A. Kleibert, A. Balan, R. V. Chopdekar, M. Wyss, J. Perron, A. Scholl, F. Nolting, and L. J. Heyderman, Phys. Rev. Lett. 111, 057204 (2013). 
[19] M. Tanaka, E. Saitoh, H. Miyajima, T. Yamaoka, and Y. Iye, Phys. Rev. B 73, 052411 (2006).

[20] Y. Qi, T. Brintlinger, and J. Cumings, Phys. Rev. B 77, 094418 (2008).

[21] E. Mengotti, L. J. Heyderman, A. Fraile, Rodríguez, A. Bisig, L. Le Guyader, F. Nolting, and H. B. Braun, Phys. Rev. B 78, 144402 (2008).

[22] S. Ladak, D. E. Read, G. K. Perkins, L. F. Cohen, and W. R. Branford, Nat. Phys. 6, 359 (2010).

[23] E. Mengotti, L. J. Heyderman, A. Fraile Rodríguez, F. Nolting, R. V. Hügli, and H. B. Braun, Nat. Phys. 7, 68 (2011).

[24] A. S. Wills, R. Ballou, and C. Lacroix, Phys. Rev. B 66, 144407 (2002).

[25] Y. Tabata, H. Kadowaki, K. Matsuhira, Z. Hiroi, N. Aso, E. Ressouche, and B. Fåk, Phys. Rev. Lett. 97, 257205 (2006).

[26] V. Kapaklis, U. B. Arnalds, A. Harman-Clarke, E. Th. Papaioannou, M. Karimipour, P. Korelis, A. Taroni, P. C. W. Holdsworth, S. T. Bramwell, and B. Hjörvarsson, New J. Phys. 14, 035009 (2012).

[27] J. M. Porro, A. Bedoya-Pinto, A. Berger, and P. Vavassori, New J. Phys. 15, 055012 (2013).

[28] S. Zhang, I. Gilbert, C. Nisoli, G. W. Chern, M. J. Erickson, L. O' Brien, C. Leighton, P. E. Lammert, V. H. Crespi, and Peter Schiffer, Nature (London) 500, 553 (2013).

[29] A. Farhan, P. M. Derlet, A. Kleibert, A. Balan, R. V. Chopdekar, M. Wyss, L. Anghinolfi, F. Nolting, and L. J. Heyderman, Nat. Phys. 9, 375 (2013).

[30] R. Milo, S. Shen-Orr, S. Itzkovitz, N. Kashtan, D. Chklovskii, and U. Alon, Science 298, 824 (2002).
[31] H. Markram, Nat. Rev. Neurosci. 7, 153 (2006).

[32] L. Grill, M. Dyer, L. Lafferentz, M. Persson, M. V. Peters, and S. Hecht, Nat. Nanotechnol. 2, 687 (2007).

[33] J. Stöhr, Y. Wu, B. D. Hermsmeier, M. G. Samant, G. R. Harp, S. Koranda, D. Dunham, and B. P. Tonner, Science 259, 658 (1993).

[34] L. Le Guyader, A. Kleibert, A. Fraile Rodríguez, S. El Moussaoui, A. Balan, M. Buzzi, J. Raabe, and F. Nolting, J. Electron Spectrosc. Relat. Phenom. 185, 371 (2012).

[35] J. Rothman, M. Kläui, L. Lopez-Diaz, C. A. F. Vaz, A. Bleloch, J. A. C. Bland, Z. Cui, and R. Speaks, Phys. Rev. Lett. 86, 1098 (2001).

[36] R. V. Chopdekar, G. Duff, R. V. Hügli, E. Mengotti, D. A. Zanin, L. J. Heyderman, and H. B. Braun, New J. Phys. 15, 125033 (2013).

[37] G. Möller and R. Moessner, Phys. Rev. B 80, 140409(R) (2009).

[38] G. W. Chern, P. Mellado, and O. Tchernyshyov, Phys. Rev. Lett. 106, 207202 (2011).

[39] L. A. Mól, R. L. Silva, R. C. Silva, A. R. Pereira, W. A. Moura-Melo, and B. V. Costa, J. Appl. Phys. 106, 063913 (2009).

[40] Z. Budrikis, K. L. Livesey, J. P. Morgan, J. Akerman, A. Stein, S. Langridge, C. H. Marrows, and R. L. Stamps, New J. Phys. 14, 035014 (2012).

[41] R. C. Silva, F. S. Nascimento, L. A. S. Mól, W. A. Moura-Melo, and A. R. Pereira, New J. Phys. 14, 015008 (2012).

[42] D. Levis, L. F. Cugliandolo, L. Foini, and M. Tarzia, Phys. Rev. Lett. 110, 207206 (2013). 